\title{
Evaluation de la qualité nutritionnelle du lait cru dans les élevages traditionnels de Kaolackau Sénégal
}

\author{
M. KALANDI, A. SOW, W.V.H. GUIGMA, M. Z. ZABRE, \\ A. BATHILY et G. J. SAWADOGO* \\ Ecole Inter-Etats des Sciences et Médecine Vétérinaires (EISMV), \\ Laboratoire d'Endocrinologie et de Radio-Immunologie, BP. 5077 Dakar-Fann, Sénégal. \\ *Auteur correspondant, E-mail: swadogo@refer.sn ; Tel. (221) 338651008 ; Fax : (221) 338254283
}

\section{RESUME}

Face à la concurrence des importations de laits et de produits laitiers, la production d'un lait local présentant de bonnes qualités nutritionnelle et sanitaire peut améliorer sa compétitivité. L'appréciation de la qualité nutritionnelle du lait cru en rapport avec les pratiques d'élevage a été faite dans 14 élevages traditionnels de la région de Kaolack (Sénégal). Au total, 120 échantillons ont été prélevés dans des flacons stériles de $30 \mathrm{ml}$. Les taux de matières grasses (MG), de matières protéiques (MP), de matière sèche (MS), de lactose ainsi que la densité ont été déterminés par spectrophotométrie (Dairy Milk Analyser, Miris AB, Uppsala, Suède). Le suivi des élevages a permis de collecter des informations épidémiologiques des élevages. La majorité des élevages étaient conduits selon un système extensif (78\%). Les races exploitées étaient composées majoritairement des Djakoré (53,6\%), le zébu Gobra (28,2\%), les métisses issues de l'insémination artificielle $(13,6 \%)$ et la N'Dama (4,6\%). La concentration des paramètres nutritionnels du lait cru était surtout influencée par le niveau de lactation et la saison. Les résultats ont montré des concentrations de 47,03 g/l en MG, 137,89 g/l en MS, 39,10 g/l en MP et 44,66 g/l en lactose ; pour le lait individuel des vaches. Quant au lait de mélange de la ferme, les valeurs moyennes de 44,97 g/l; 134,28 g/l; 35,09 g/l; 45,69 g/l ont été respectivement trouvés pour les mêmes composants du lait. La densité à $+20{ }^{\circ} \mathrm{C}$ était de 1,029 pour le lait de mélange et le lait individuel des vaches. Le lactose était corrélé à la race et au niveau de lactation $(p<0,05)$ tandis que les teneurs en MG, MS, MP étaient corrélées uniquement au niveau de lactation $(p<0,05)$. Cette étude a montré que le lait au pis est de bonne qualité sur le plan nutritionnel. Cependant, la vulgarisation des bonnes pratiques d'hygiène tout au long de la chaîne de production est nécessaire pour l'amélioration de la qualité du lait cru.

(C) 2015 International Formulae Group. All rights reserved.

Mots clés: Lait, nutritionnelle, compétitivité, Kaolack, Sénégal.

\section{INTRODUCTION}

Au Sénégal, avec une contribution à hauteur de $7,4 \%$ au produit intérieur brut (PIB), l'élevage est la deuxième activité la plus importante du secteur primaire après l'agriculture (MEF/DPS, 2004). La production de lait est essentiellement assurée par un cheptel bovin de 3,313 millions de têtes (DIREL, 2010).

Le lait et les produits laitiers appartiennent aux habitudes alimentaires de nombreuses civilisations. Composante 
essentielle du régime alimentaire des populations pastorales ou agropastorales et aussi une source de revenu, le lait est une importante source de protéines pour les populations de l'Afrique de l'Ouest. Au Sénégal, il représente $14 \%$ de l'apport protéique de la population (DASP, 2010).

Le Sénégal, est un pays à tradition pastorale avec un effectif important en bovins. Mais, compte tenu de sa rapide croissance démographique et son urbanisation, la production locale du lait ne couvre pas les besoins des populations. Le Sénégal dépend des marchés extérieurs pour les deux tiers de son approvisionnement en lait (DASP, 2010), essentiellement sous la forme de poudre de lait. Ces importations de laits et de produits laitiers coûtent chers au pays (60 milliards de francs CFA en 2011) (ME, 2011) et détruisent les emplois ruraux des pasteurs.

Pour sauver les emplois ruraux, il faut rendre le lait local compétitif. A ce niveau, plusieurs tentatives ont été testées ou sont en cours. Parmi les tentatives les plus prometteuses, il y a l'insémination artificielle, la complémentation alimentaire et la maîtrise de l'hygiène et de la qualité du lait produit.

Ces trois volets ont été peaufinés selon l'approche du concept IAR4D (Recherche Agricole Intégrée pour le Développement) dans le cadre du projet Appui à l'amélioration durable de la productivité et de la compétitivité des filières laitières bovines en Afrique de l'Ouest et du Centre (AMPROLAIT) implémenté au Sénégal, au Niger, au Tchad, au Burkina Faso et au Cameroun.

Par ailleurs dans une grande partie de l'Afrique et en particulier au Sénégal, la production laitière est dominée par le secteur informel à travers de petites exploitations qui ont des pratiques traditionnelles de production. La traite manuelle du lait, les conditions de transport et le maintien de la chaîne de froid le long de la filière sont susceptibles de conduire à un lait de qualité hygiénique douteuse. En effet, de nombreux auteurs ont rapporté qu'au Burkina Faso
(Savadogo et al., 2004 ; Millogo et al., 2008 ; 2010), au Mali (Bonfoh et al., 2003; 2006) et au Ghana (Donkor et al., 2007), le lait local est de mauvaise qualité hygiénique tout au long de la chaîne de production, de la ferme à la table. Le lait, qu'il soit frais ou transformé, est un excellent milieu de culture pour plusieurs microorganismes avec pour résultante l'altération du produit ou les infections/intoxications chez les consommateurs (Murinda et al., 2004 ; Olivier et al., 2005).

De nombreuses études ont par ailleurs montré que la qualité du lait dépend des vaches (Pougheon et Goursaud, 2001; Millogo, 2010), de l'alimentation (Whitlock et al., 2003), du stade de lactation (Kay et al., 2005), de la traite (Millogo, 2010) et bien d'autres facteurs telles que la santé de l'animal (Forsbäck, 2010) et les conditions générales d'élevage (Gran et al., 2002 ; Sraïri et al., 2009). En plus, la composition du lait cru a une influence significative sur la transformation et la qualité du produit final (Forsbäck, 2010).

La zone de Kaolack étant une zone pastorale par excellence où il $\mathrm{y}$ a de nombreux élevages traditionnels, il est important d'évaluer la qualité nutritionnelle des laits qu'ils produisent afin d'orienter les consommateurs, ce qui pourra booster la filière laitière locale.

C'est dans ce contexte que cette étude s'est réalisée avec pour principal objectif d'évaluer la qualité nutritionnelle du lait cru en rapport avec les pratiques d'élevage en zone périurbaine de Kaolack. De façon spécifique, il s'agissait de caractériser les élevages laitiers traditionnels, d'évaluer la qualité nutritionnelle du lait cru et déterminer l'effet des facteurs d'élevages sur la qualité nutritionnelle du lait cru.

\section{MATERIEL ET METHODES \\ Zone d'étude}

La présente étude a été menée dans la région de Kaolack située dans le bassin arachidier entre les $14^{\circ} 30$ et $16^{\circ} 30$ Ouest, et 
$13^{\circ} 30$ et $1^{\circ} 30$ Nord. Le climat y est caractérisé par une saison pluvieuse d'une durée variable (juillet - octobre) avec une pluviométrie moyenne annuelle est de 400 mm et d'une saison sèche (novembre à juin). Les températures varient entre $15^{\circ} \mathrm{C}$ et $40^{\circ} \mathrm{C}$. La population, en majorité rurale, s'intéresse aux activités du secteur primaire axées sur l'agriculture, l'élevage, la pêche et la foresterie. C'est une zone d'élevage par excellence et les races élevées sont les zébus Gobra, les Djakoré, les taurins Ndama, et de nombreux métis issus des croisements entre races locales et exotiques (Figure 1).

\section{Echantillonnage}

Quatorze élevages traditionnels de la zone périurbaine de Kaolack (Sénégal) ont constitué la base d'échantillonnage. Les prélèvements ont été effectués au pis et au mélange dans des flacons de $30 \mathrm{ml}$. Seul le lait du matin a été prélevé. Au total, 120 échantillons ont été prélevés et placés dans une glacière munie de conservateurs de glace.
Une enquête de terrain a permis de recueillir des informations destinées à identifier et à caractériser les élevages ainsi que leur mode de conduite.

\section{Détermination des paramètres nutritionnels du lait cru}

Les échantillons ont été analysés 2 à 3 heures maximum après le prélèvement. Les taux de matières grasses (MG), de matières protéiques (MP), de matière sèche (MS), de lactose ainsi que la densité ont été déterminés par spectrophotométrie (Dairy Milk Analyser, Miris AB, Uppsala, Suède).

\section{Analyses statistiques}

Les données ont été traitées dans STATA SE9. $2^{\circledR}$. Le test de Student (t-test) et ANOVA ont été utilisés pour comparer les teneurs moyennes de MG, MP, MS et lactose obtenues entre des groupes de vaches donnés. La différence entre les valeurs d'un paramètre a été considérée statistiquement significative à $\mathrm{p}<0,05$.

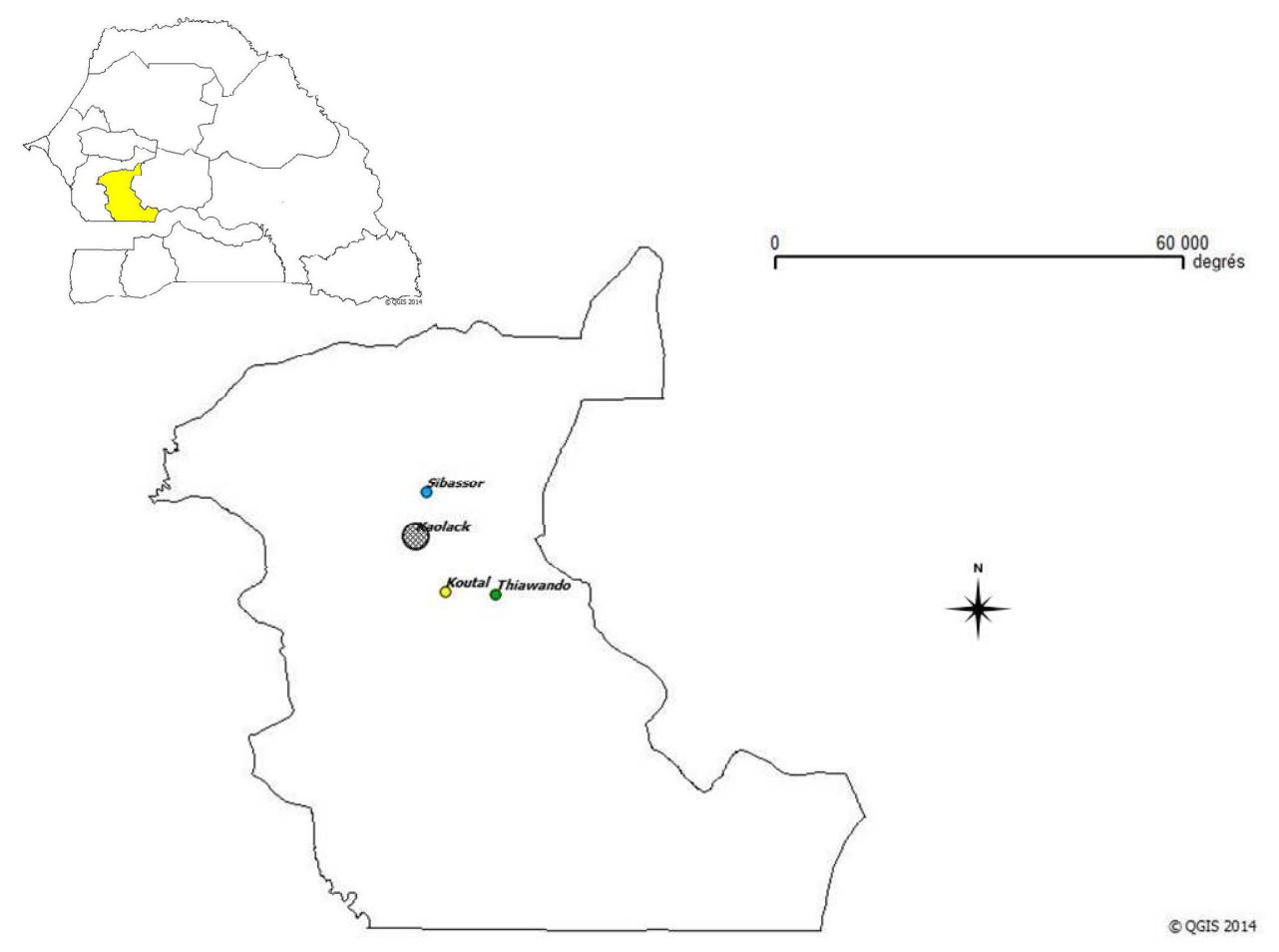

Figure 1: Carte de la région de Kaolack. 


\section{RESULTATS}

Caractérisation des élevages laitiers

Les principales races exploitées dans les élevages enquêtés étaient le Djakoré (53,6\%), le zébu Gobra (28,2\%), les métisses issues de l'insémination artificielle $(13,6 \%)$ et la N'Dama $(4,6 \%)$. Le Tableau 1 présente la répartition des vaches par tranches d'âge et le nombre de mois de lactation.

\section{Paramètres nutritionnels du lait cru}

Le Tableau 2 présente la concentration moyenne des paramètres nutritionnels du lait individuel et du lait de mélange. L'analyse statistique n'a pas révélé de différence significative entre les deux types de lait $(\mathrm{p}>0,05)$. Toutes les valeurs des paramètres nutritionnels obtenues dans les échantillons de lait des élevages traditionnels de Kaolack étaient dans la fourchette des valeurs de référence à l'exception de la densité qui apparaît relativement faible.
Effet des facteurs d'élevages sur la qualité nutritionnelle du lait cru

Race

La concentration en lactose des prélèvements a varié significativement selon la race $(\mathrm{p}<0,05)$ (Tableau 3). Elle était faible dans le lait des vaches N'Dama (29,2 g/l).

Nombre de mois de lactation

Les concentrations moyennes des paramètres nutritionnels moyens du lait cru selon le nombre de mois de lactation sont présentées dans le Tableau 4. On observe une augmentation significative $(\mathrm{p}<0,05)$ de la concentration en MG, MP, MS et du lactose jusqu'au dixième mois de lactation. Cependant, la variation de la densité n'est pas significative.

Age

Il en ressort que les concentrations du lait cru en MG, MP, MS et lactose ainsi que la densité ne varient pas significativement $(\mathrm{p}>$ 0,05) en fonction de l'âge de la vache (Tableau 5).

\section{Saison}

Le Tableau 6 montre les variations de la concentration des paramètres nutritionnels du lait cru suivant la saison. L'analyse statistique a révélé une diminution significative $(p<0,05)$ en saison chaude de la concentration en MG, MP et du lactose.

Tableau 1: Répartition des vaches par tranches d'âge et nombre de mois de lactation.

\begin{tabular}{lccccc}
\hline Tranches âge (an) & {$[3-5]$} & {$[6-8]$} & {$[9-11]$} & {$[12-14]$} & Total \\
Effectif & 23 & 46 & 27 & 14 & 110 \\
Nombre de mois de lactation & {$[2-4]$} & {$[5-7]$} & {$[8-10]$} & {$[11-13]$} & \\
Effectif & 19 & 47 & 35 & 9 & 110 \\
\hline
\end{tabular}

Tableau 2: Teneur des paramètres nutritionnels moyens et les écarts type du lait individuel et du lait de mélange.

\begin{tabular}{|c|c|c|c|c|}
\hline Paramètres & $\begin{array}{l}\text { Lait ind. } \\
n=110\end{array}$ & $\begin{array}{l}\text { Lait de mél. } \\
\qquad n=10\end{array}$ & p-value & $\begin{array}{l}\text { Valeur de } \\
\text { référence }\end{array}$ \\
\hline Matière grasse $(\mathrm{g} / \mathrm{l})$ & $47,03 \pm 10,5$ & $44,97 \pm 8,9$ & 0,77 & $24,72-56,82$ \\
\hline Matière sèche (g/l) & $137,89 \pm 12,7$ & $134,28 \pm 11,4$ & 0,65 & $117,42-141,52$ \\
\hline Matière protéique $(\mathrm{g} / \mathrm{l})$ & $39,10 \pm 2,9$ & $35,09 \pm 3,9$ & 0,14 & $29,87-51,65$ \\
\hline Lactose $(\mathrm{g} / \mathrm{l})$ & $44,66 \pm 6,6$ & $45,69 \pm 7,4$ & 0,61 & $37,08-57,85$ \\
\hline Densité à $20^{\circ} \mathrm{C}$ & $1,029 \pm 0,00$ & $1,029 \pm 0,00$ & 0,96 & $1,030-1,033$ \\
\hline
\end{tabular}

ind: individuel; mél.: mélange. 
Tableau 3: Variation des paramètres nutritionnels moyens du lait frais suivant la race.

\begin{tabular}{lccccc}
\hline Paramètres & $\begin{array}{c}\text { Djakoré } \\
\mathbf{n = 5 9}\end{array}$ & $\begin{array}{c}\text { Gobra } \\
\mathbf{n = 3 1}\end{array}$ & $\begin{array}{c}\text { N'Dama } \\
\mathbf{n = 5}\end{array}$ & $\begin{array}{c}\text { Métisse } \\
\mathbf{n = 1 5}\end{array}$ & p-value \\
\hline Matière grasse $(\mathrm{g} / \mathrm{l})$ & $43,26 \pm 21,1$ & $53,04 \pm 10,8$ & $60,09 \pm 4,6$ & $45,23 \pm 6,8$ & 0,116 \\
Matière sèche $(\mathrm{g} / \mathrm{l})$ & $137,1 \pm 36,7$ & $141,14 \pm 21,2$ & $148,99 \pm 34,4$ & $131,2 \pm 21,7$ & 0,459 \\
Matière protéique & $39,24 \pm 4,6$ & $38,76 \pm 2,2$ & $40,65 \pm 3,3$ & $38,69 \pm 6,4$ & 0,966 \\
$(\mathrm{~g} / \mathrm{l})$ & $46,35 \pm 4,4$ & $41,94 \pm 5,6$ & $29,22 \pm 1,13$ & $34,99 \pm 6,9$ & $0,001^{*}$ \\
Lactose $(\mathrm{g} / \mathrm{l})$ & $1,030 \pm 0,00$ & $1,028 \pm 0,00$ & $1,029 \pm 0,01$ & $1,029 \pm 0,00$ & 0,628 \\
Densité à 20 ${ }^{\circ} \mathrm{C}$ & & & &
\end{tabular}

Tableau 4 : Variation des paramètres nutritionnels moyens du lait frais suivant le niveau de lactation.

\begin{tabular}{lccccc}
\hline Paramètres & {$[\mathbf{2}-\mathbf{4}]$} & {$[\mathbf{5}$-7] } & {$[\mathbf{8}$-10] } & {$[\mathbf{1 1 - 1 3 ]}$} & $\boldsymbol{p}$-value \\
\hline Matière grasse $(\mathrm{g} / \mathrm{l})$ & $37,90 \pm 12,7$ & $46,51 \pm 17,2$ & $52,99 \pm 22,8$ & $52,33 \pm 9,1$ & $0,039^{*}$ \\
Matière protéique $(\mathrm{g} / \mathrm{l})$ & $36,87 \pm 15,9$ & $41,67 \pm 9,8$ & $46,92 \pm 13,8$ & $40,7 \pm 1,12$ & $0,000^{* *}$ \\
Matière sèche $(\mathrm{g} / \mathrm{l})$ & $125,66 \pm 22,1$ & $135,83 \pm 23,6$ & $145,91 \pm 30,9$ & $144,33 \pm 22,7$ & $0,007 *$ \\
Lactose $(\mathrm{g} / \mathrm{l})$ & $45,22 \pm 4,6$ & $45,48 \pm 18,3$ & $43,22 \pm 10,0$ & $40,1 \pm 10,7$ & $0,002^{*}$ \\
Densité à $20{ }^{\circ} \mathrm{C}$ & $1,030 \pm 0,00$ & $1,029 \pm 0,00$ & $1,029 \pm 0,00$ & $1,028 \pm 0,01$ & 0,860 \\
\hline
\end{tabular}

* Différence significative $\mathrm{p}<0,05$; ** Différence très significative.

Tableau 5: Variation des paramètres nutritionnels moyens du lait frais suivant l'âge.

\begin{tabular}{lccccc}
\hline Paramètres & {$[\mathbf{3 - 5}]$} & {$[\mathbf{6 - 8}]$} & {$[\mathbf{9 - 1 1}]$} & {$[\mathbf{1 2 - 1 4 ]}$} & $\boldsymbol{p}$-value \\
\hline Matière grasse $(\mathrm{g} / \mathrm{l})$ & $46,4 \pm 17,2$ & $43,22 \pm 19,9$ & $48,47 \pm 12,8$ & $49,99 \pm 12,7$ & 0,694 \\
Matière protéique $(\mathrm{g} / \mathrm{l})$ & $40,72 \pm 13,6$ & $40,95 \pm 12,9$ & $40,77 \pm 19,72$ & $41,94 \pm 21,1$ & 0,987 \\
Matière sèche $(\mathrm{g} / \mathrm{l})$ & $137,85 \pm 30,7$ & $132,84 \pm 29,9$ & $137,72 \pm 28,55$ & $141,52 \pm 32,0$ & 0,384 \\
Lactose $(\mathrm{g} / \mathrm{l})$ & $44,85 \pm 19,1$ & $44,65 \pm 13,2$ & $43,13 \pm 20,74$ & $42,35 \pm 20,0$ & 0,439 \\
Densité à $20^{\circ} \mathrm{C}$ & $1,031 \pm 0,01$ & $1,029 \pm 0,0$ & $1,027 \pm 0,02$ & $1,033 \pm 0,0$ & 0,054 \\
\hline
\end{tabular}

Tableau 6: Variation des paramètres nutritionnels moyens du lait frais suivant la saison.

\begin{tabular}{llll}
\hline Paramètres & Saison froide & Saison chaude & p-value \\
\hline Matière grasse $(\mathrm{g} / \mathrm{l})$ & $50,52 \pm 23,04$ & $39,99 \pm 20,3$ & $0,016^{*}$ \\
Matière sèche $(\mathrm{g} / \mathrm{l})$ & $138,92 \pm 21,3$ & $141,58 \pm 28,3$ & 0,154 \\
Matière protéique $(\mathrm{g} / \mathrm{l})$ & $42,5 \pm 6,6,9$ & $38,77 \pm 10,0$ & $0,033^{*}$ \\
Lactose $(\mathrm{g} / \mathrm{l})$ & $45,38 \pm 5,0$ & $42,05 \pm 5,3$ & $0,002^{*}$ \\
Densité à $20{ }^{\circ} \mathrm{C}$ & $1,029 \pm 0,00$ & $1,023 \pm 0,01$ & 0,522 \\
\hline \multicolumn{2}{c}{${ }^{\circ}$ significatif à $\mathrm{p}<0,05}$. & &
\end{tabular}




\section{DISCUSSION \\ Caractérisation des élevages laitiers traditionnels}

La principale race exploitée $(53,6 \%)$ est le Djakoré suivie de la race Gobra $(28,2 \%)$. Les travaux de Nkolo (2009) à Thiès et d'Asseu (2010) à Kaolack ont montré que c'était la race Gobra qui prédominait dans les élevages avec respectivement $82 \%$ et $86,4 \%$ des effectifs du cheptel. En effet, le Djakoré a hérité de la rusticité de la race Gobra et la trypanotolérance de la race N'Dama, il assure aussi bien la traction dans les champs qu'une production laitière par rapport aux autres races locales. Il faut aussi noter le nombre de plus en plus croissant des métisses dans les élevages de Kaolack par le biais de l'insémination artificielle pratiquée depuis une quinzaine d'années.

\section{Paramètres nutritionnels du lait cru}

Il existe une corrélation positive entre le taux de MG et la concentration en protéine $\mathrm{du}$ lait. Les taux similaires moyens de MG ont été obtenus dans les fermes autour de Ouagadougou au Burkina Faso (Sidibé-Anago et al., 2006 ; Millogo et al., 2009; Millogo, 2010). Il faut souligner que tous ces résultats étaient dans l'intervalle des normes (24 à 55 $\mathrm{g} / \mathrm{l})$ pour la $\mathrm{MG}$ et $(29$ à $50 \mathrm{~g} / \mathrm{l})$ pour la MP selon la norme NS 03 - 020-Lait cru. La concentration de la $\mathrm{MG}$ dans le lait variait essentiellement en fonction de l'alimentation et du niveau de production.

La concentration en MS du lait individuel et le lait de mélange étaient de $137,89 \mathrm{~g} / \mathrm{l}$ et 134,28 g/l respectivement. Ce résultat reste supérieur à ceux de Labioui et al. (2009) $(117,5 \mathrm{~g} / \mathrm{l})$ et de Sboui et al. (2009)(104,8) au Maroc et en Tunisie respectivement. Ces auteurs ont travaillé sur des vaches hautes productrices (Montbéliarde et Holstein). Comme la MG, la teneur du lait frais en MS dépend de l'alimentation, du climat, mais également de la race.

La valeur moyenne de lactose du lait individuel et le lait de mélange était de 44,66 $\mathrm{g} / 1$ et 45,69 g/l respectivement. Ce résultat était similaire à ceux obtenus au Maghreb par (Labioui et al., 2009 ; Sboui et al., 2009). Ces valeurs sont relativement inférieures à la valeur moyenne de référence qui est de $49 \mathrm{~g} / \mathrm{l}$. Le lactose, principal sucre présent dans le lait est le substrat de fermentation pour les bactéries lactiques (Streptococcus, Lactobacillus, Enterococcus, Leuconostoc et Aerococcus). Ces bactéries se caractérisent par leur aptitude à fermenter le lactose avec production d'acide lactique. Dans cette étude, les échantillons étaient analysés 3 heures après la traite, ce qui aurait favorisé le développement des bactéries lactiques, d'où la teneur faible en lactose.

La valeur moyenne de la densité à 20 ${ }^{\circ} \mathrm{C}$ du lait individuel et du lait de mélange était de 1,029. Ce résultat est comparable à celui de Labioui et al. (2009) au Maroc (1,030). La valeur normale de la densité est comprise entre 1,030 et 1,033 pour un lait individuel, et 1,020 à 1,038 pour le lait de mélange à $20^{\circ} \mathrm{C}$. En effet, la densité diminue en cas de mouillage du lait, ce qui n'est pas le cas dans cette étude, car les échantillons étaient prélevés au pis. La faible densité du lait individuel pourrait être due à des facteurs tels que la teneur en MS, MG, l'augmentation de la température et l'alimentation (Labioui et al., 2009).

\section{Effet des facteurs d'élevage sur la qualité nutritionnelle du lait frais}

La concentration en lactose varie significativement dans le lait cru selon la race. L'effet de la race sur la qualité nutritionnelle du lait a été démontré par plusieurs auteurs. En effet, la Holstein, vache laitière haute productrice, produirait une quantité importante de lait avec une faible concentration en matières utiles (MG, MP) (Matallah et al., 2015). En effet, la concentration en matières utiles est inversement corrélée avec le rendement de lait. Notons que dans cette étude, la production était faible et il n'y avait pas une 
grande différence entre la quantité de lait produite par les différentes races, ce qui justifierait l'absence de variation des autres paramètres nutritionnels.

De nos résultats, il ressort que l'âge de la vache n'avait aucune influence sur la teneur des paramètres nutritionnels de son lait. La concentration des paramètres nutritionnels était surtout influencée par le nombre de mois de lactation. Une augmentation significative de la concentration en MG, MP, MS et du lactose a été observée à partir du deuxième mois jusqu'au dixième mois de lactation. Millogo (2010) a également observé au Burkina Faso, une augmentation progressive de la MG après le premier mois de lactation jusqu'au tarissement. Cette variation peut être due à l'effet conjugué de deux hormones, la prolactine et la GH (hormone de croissance). Ces hormones ont un effet à long terme sur la production et la composition du lait (augmentation du taux butyreux et baisse du taux protéique).

Cette étude indique que la concentration du lait cru en MG, MP et en lactose diminuait significativement en saison chaude. La concentration en MG passe de 50,52 g/l en saison froide à $39,99 \mathrm{~g} / \mathrm{l}$ en saison chaude, la MP de 42,5 g/l en saison froide à 38,77 g/l en saison chaude, et le lactose de $45,38 \mathrm{~g} / \mathrm{l}$ en saison froide à 42,05 g/l en saison chaude. Ce phénomène a été également observé à N'Djamena au Tchad où une variation a été notée dans la concentration des paramètres nutritionnels du lait selon la saison (Koussou et al., 2009). En effet, ces auteurs ont observé une variation non significative de la $\mathrm{MG}$, par contre la MS passe de 124,9 g/l en saison froide à 118,3 $\mathrm{g} / \mathrm{l}$ en saison chaude. Ces variations s'expliquent surtout par la disponibilité alimentaire. Dans la région de Kaolack, la saison chaude (mars à juin), correspond à la période de soudure, où on assiste à un appauvrissement du pâturage et à un épuisement des stocks alimentaires. Les éleveurs ne disposent pas des moyens nécessaires pour assurer l'alimentation du troupeau. C'est la raison pour laquelle, une baisse de la qualité nutritionnelle du lait cru est observée durant cette période.

\section{Conclusion}

L'analyse de la qualité du lait cru au niveau des élevages traditionnels a montré que le lait au pis est de bonne qualité sur le plan nutritionnel. Au regard de ces résultats une nouvelle investigation sur la qualité nutritionnelle et microbiologique des laits crus des fermes, des collecteurs et des vendeurs de la région de Kaolack pourra rendre les produits laitiers locaux compétitifs car la maîtrise de la qualité tout au long de la filière reste un défi: une marque de qualité d'abord, ensuite la définition d'un label et enfin un code de bonnes pratiques et d'hygiène adapté au contexte local.

\section{REMERCIEMENTS}

Nous remercions le Conseil Ouest et Centre africain pour la Recherche et le Développement Agricole (CORAF) pour avoir financé cette étude à travers le projet Appui à l'amélioration durable de la productivité et de la compétitivité des filières laitières bovines en Afrique de l'Ouest et du Centre (AMPROLAIT). Nos remerciements vont également à l'endroit de la coordination régionale dudit projet, des éleveurs et de tout le personnel du Laboratoire d'Endocrinologie et de Radio-immunologie de l'EISMV de Dakar pour leur disponibilité.

\section{REFERENCES}

Asseu KC. 2010. Evaluation du degré d'acceptation de l'insémination artificielle bovine à Kaolack au Sénégal. Thèse: Med. Vét., Dakar, 92p.

Bonfoh B, Roth C, Traore AN, Fané A, Simbe CF, Alfaroukh IO, Nicolet J, Farah Z Zinsstag J. 2006. Effect of washing and disinfecting containers on the microbiological quality of fresh milk sold in Bamako (Mali). Food Control, 17: 153-161.

Bonfoh B, Wasem A, Traore AN, Fané A, Spillmann H, Simbé CF, Alfaroukh IO, 
Nicolet J, Farah Z Zinsstag J. 2003. Microbiological quality of cows' milk taken at different intervals from the udder to the selling point in Bamako (Mali). Food Control, 14: 495-500.

Donkor ES, Aning KG, Quaye J. 2007. Bacterial contaminations of informally marketed raw milk in Ghana. Ghana Med. J., 41: 58-61.

Dufour B, De Buyser ML, Brisabois A, Espié E, Delmas G. 2005. La sécurité microbiologique : Implication du lait et des produits laitiers dans les maladies infectieuses d'origine alimentaire. In $L a$ Sécurité des Produits Laitiers, 5ème Conférence Européenne d'Arilait (CREAL 2004). Editeur Arilait Recherches : Paris ; 13-21.

Duteurtre G, Dieye PN, Dia D. 2005. L'impact des importations de volailles et de produits laitiers sur la production locale au Sénégal, Etudes et documents «Ouverture des frontières et développement agricole dans les pays de l'UEMOA», ISRA - BAME, Dakar ; $78 \mathrm{p}$.

Forsbäck L, Lindmark-Mansson H, Andrén A, Akerstedt M, Andrée L, SvennerstenSjaunja K. 2010. Day-to-day variation in milk yield and milk composition at the udder-quarter level. J. Dairy Sci., 93: 3569-3577.

Gran HM, Mutukumira AN, Wetlesen A, Narvhus JA. 2002. Smallholder dairy processing in Zimbabwe: hygienic practices during milking and the microbiological quality of the milk at the farm and on delivery. Food Control, 13: 41-47.

Kay JK, Weber WJ, Moore CE, Bauman DE, Hansen LB, Chester-Jones H, Crooker BA, Baumgard LH. 2005. Effects of weak of lactation and genetic selection for milk yield on milk fatty acid composition in Holstein cows. J. Dairy Sci., 88: 3886-3893.

Koussou MO, Grimaud P, Mopaté LY. 2009. Evaluation de la qualité physico-chimique et hygiénique du lait de brousse et des produits laitiers locaux commercialisés dans les bars laitiers de N'Djamena au Tchad. Rev. Élev. Méd. Vét. Pays Trop., 60(1-4): 45-49.

Labioui H, Elmoualdi L, Benzakour A, Yachioui ME, Berny EH, Ouhssine M. 2009. Etude physicochimique et microbiologique de laits crus. Bull. Soc. Pharm. Bordeaux, 148: 7-16.

Matallah S, Bouchelaghem S, Matallah F. 2015. Variations de la composition chimique du lait de vache Holstein dans le nord-est de l'Algérie. Livest. Res. Rural Dev., 27 Article \#16. Retrieved February 6, 2015, from http://www.lrrd.org/lrrd27/1/mata27016.h tml.

Millogo V, Ouédraogo GA, Agenäs, S, Svennersten-Sjaunja K. 2008. Survey on dairy cattle milk production and milk quality problems in peri-urban areas in Burkina Faso. Afr. J. Agric. Res., 3(3): 215-224.

Millogo V, Ouédraogo GA, Agenäs, S, Svennersten-Sjaunja K. 2009. Day-to-day variation in yield, composition and somatic cell counts of saleable milk in hand-milked zebu dairy cattle. Afr. J. Agric. Res., 4(3): 151-155.

Millogo V, Svennersten Sjaunja K, Ouédraogo GA, Agenäs S. 2010. Raw milk Hygiene at farms, processing units and local markets in Burkina Faso. Food Control, 21: 1070-1074.

Murinda SE, Nguyen LT, Nan HM, Almeida RA, Headrick SJ, Oliver SP. 2004. Detection of sorbitol-negative and sorbitol-positive Shiga toxin-producing Escherichia coli, Listeria monocytogenes, Campylobacter jejuni, and Salmonella spp. in dairy farm environmental samples. Foodborne Pathog. Dis., 1: 97-104.

Nkolo S. 2009. Typologie des élevages bovins pratiquant l'insémination artificielle en milieu traditionnel au Sénégal : Cas de la Région de Thiès. Mémoire Master : 
Productions Animales et Développement Durable, Dakar, 30p.

Oliver SP, Jayarao BM Almeida RA. 2005. Foodborne pathogens in milk and the dairy environment: food safety and public health implications. Foodborne Pathog. Dis., 2(11): 15-29.

Pougheon S, Goursaud J. 2001. Le lait caractéristiques physicochimiques. In Lait, Nutrition, Santé, DEBRY G (ed). Tec et Doc : Paris ; 2-42.

Savadogo A, Ouattara CAT, Savadogo PW, Ouattara AS, Barro N, Traoré AS. 2004. Microorganisms Involved in Fulani Traditional Fermented Milk in Burkina Faso. Pak. J. Nutr., 3(2): 134-139.

Sboui A, Khorchani T, Djegham M, Belhadj O. 2009. Comparaison de la composition physicochimique du lait camelin et bovin $\mathrm{du}$ Sud tunisien; variation du $\mathrm{pH}$ et de l'acidité à différentes températures. Afr. Sci., 05(2): 293 - 304.

DIREL 2010. Statistiques d'élevage en 2010. Ministère de l'Elevage, Dakar, Sénégal, $52 \mathrm{p}$.

ME. 2011. Rapport annuel du Ministère de l’Elevage, Dakar, Sénégal, 68p.
MEF/DPS. 2004. Situation Economique et Sociale du Sénégal. Ministère de l'Economie et des Finances / Division de la Prévision et de la Statistique, Dakar, Sénégal, 175p.

DASP. 2010. Créneau porteur du secteur secondaire: production de lait local. Ministère de l'Economie et des Finances/ Direction de l'Appui au Secteur Privé Dakar, Sénégal, 4p.

Sidibé-Anago AG, Ouédraogo GA, Ledin I. 2006. Effect of partly replacing cottonseed cake with Mucuna spp. (Var Ghana) hay on feed intake and digestibility, milk yield and milk composition of zebu cows. Trop. Anim. Health Prod., 38: 563-570.

Sraïri MT, Benhouda H, Kuper M, Le Gal PY. 2009. Effect of cattle management practices on raw milk quality on farms operating in two-stage dairy chain. Trop. Anim. Health Prod., 41: 259-272.

Whitlock LA, Schingoethe DJ, Hippen AR, Kalscheur KF. AbuGhazaleh AA. 2003. Milk production and composition from cows fed high oil or conventional corn at two forage concentrations. J. Dairy Sci., 86: $2428-2437$. 